\title{
Abnormal uterine bleeding
}

\author{
Dr. Astha Saheta, Dr.C.Hariharan, Dr.Urvashi Sharma
}

\section{Introduction}

Abnormal uterine bleeding is the commonest presenting symptom and major gynecological problem re sponsible for as many as one-third of all out patient gynecologic visit.1,2 The endometrium which lines the uteri ne cavity is one of the most dynamic tissues in the human body. It is characterized by cyclic processes of cell pr oliferation, differentiation and death in response to sex steroids elaborated in the ovary. 3 Menorrhagia affects 10 $-30 \%$ of menstruating women at any one time, and may occur at some time during the perimenopause in upto 50 $\%$ of women. 4

Abnormal uterine bleeding is defined as any bleeding pattern that differs in the frequency, duration and amount from a pattern observed during a normal menstrual cycle or menopause. It is a common problem having a long list of causes in different age groups. 5 The prevalence of abnormal uterine bleeding in reproductive age group ranges from $9 \%$ to $30 \% .6$

It is a debiliating disorder both medically and socially. In addition, it is the commonest cause of iron de ficiency in the developed world and of chronic illness in the developing world (Royal College of Obstetrics and Gynaecology [RCOG], 1998). The number of menses experienced by women in their lifetimes increased as a res ult of the reduction of family size leading to shorter periods of childbearing and lactational amenorrhoea. As a c onsequence, abnormal menstruation is especially a problem of the twentieth century.7

Menstrual history and physical examination are the mainstay of evaluation of cases. Laboratory tests, $\mathrm{i}$ maging studies and histological examinations may be indicated, as well. Its management is complicated and vari able according to the case.

\section{Endometrial Sampling}

Endometrial sampling provides tissue for histological diagnosis and thus exclusion of premalignant or malignant disease. Dilatation and curettage (D\&C) has been the traditional gold-standard investigation for AUB. Alternative methods have been sought as a D\&C requires general anaesthesia and is a blind procedure for tissue sampling. Two-thirds of women examined by this method have been reported to have less than half the uterine cavity sampled and $16 \%$ less than one-quarter. The main disadvantage of a blind endometrial biopsy is that focal lesions such as polyps, submucous fibroids and localized pathological lesions may be missed. The endometrial sampling is chosen to evaluate abnormal uterine bleeding because it has several advantages over other diagnosti c methods. The hormonal assay is very expensive and laboratories with hormonal assay are not available in rural areas.

\section{Ultrasound}

Pelvic ultrasound is the least invasive of the outpatient techniques that can be used for visualisation of $t$ he structure of the uterus and for visualisation of the thickness of the endometrium.USG has a reduced diagnosti c specificity. A subsequent meta-analysis (pooling data from four studies) demonstrated that a negative ultrasou nd result of $5 \mathrm{~mm}$ or less reduced the risk of disease by $84 \%$. In contrast, in the premenopausal woman the role $\mathrm{f}$ or pelvic ultrasound is in the diagnosis of structural abnormalities as an explanation for AUB, such as submucou $\mathrm{s}$ fibroids or endometrial polyps. The advantages of pelvic ultrasound are that it is non-invasive, there is no need for anaesthesia and, in certain cases, it provides additional information that may assist both diagnosis and treat ment choice. In clinical practice it is common for radiologists and radiographers to undertake ultrasound investi gation by both methods, starting with abdominal, which requires a full bladder to provide optimal visualisation o $\mathrm{f}$ the ovaries and uterine structure.

\section{Need For Study}

In ambulatory assessment of women with abnormal uterine bleeding, the main aim is to exclude endom etrial pathology, particularly carcinoma and hyperplasia. However there is no consensus as to which set of inves tigations should be used. While some clinicians resort to ultrasound imaging techniques as well as tissue sampli $\mathrm{ng}$, others advocate endoscopic examination. There is a lack of good quality research evidence on the accuracy o $\mathrm{f}$ the various diagnostic tests in predicting endometrial lesions. This dearth of relevant evidence prompted the res earch presented in this thesis. 
1. To study clinical presentation AUB

\section{Aim And Objectives Of The Study:}

2. To study the risk Factors associated with AUB.

3. To compare the histopathology and ultrasonography features in the diagnosis of AUB.

4. To know the prevalence of AUB in patients attending gynac OPD in AcharyaVinobhaBhave Rural Hospita 1 (AVBRH)

New Classification System Categorizes Causes of Abnormal Uterine Bleeding41 From FIGO (Medscape Educa tion Clinical Briefs)

- After a thorough 5-year review process beginning with workshops in 2005, a group of clinician-investi gators from 17 countries on 6 continents who had substantial experience in AUB research developed and revised a draft system that was distributed for comments. The PALM-COEIN classification system was then discussed at a meeting held in association with the 2009 FIGO World Congress in Cape Town, South Africa, and subseque ntly approved by the FIGO Executive Board as a FIGO classification system.

- $\quad$ June 7, 2011 - The International Federation of Gynecology and Obstetrics (FIGO) has approved a ne w classification system (PALM-COEIN) for causes of abnormal uterine bleeding (AUB) in no gravid women of reproductive age.

- Out of the 9 categories in the new FIGO classification system (PALM-COEIN), the first 4 are defined a s visually objective structural criteria (PALM: polyp, adenomyosis, leiomyoma, and malignancy and hyperplasia ). The second 4 are unrelated to structural abnormalities (COEI: coagulopathy, ovulatory dysfunction, endometri al, and iatrogenic), and the final category is for entities that are not yet classified (N).

Aub: Palm Coein Classification29,30,31,42

- PALM

- $\quad$ P: Endometrial polyp

- A: Adenomyosis

- L: Leiomyoma

- M: Malignancy and hyperplasia

Coein:

- C: Coagulopathy Clotting factor deficiency or defect

- O: Ovulatory

- E: Endometrial

- I: Iatrogenic Conditions

- $\quad$ N: Not Classified

Causes of AUB by Age Group42

Reproductive age

1. Anovulation

2. Pregnancy

3. Endocrine Disorder

4. Polyps/ fibroids/ Adenomyosis

5. Medication related (oral contraceptives)

6. Infection

7. Sarcoma, ovarian

8. Coagulation disorder

Perimenopausal

1. Anovulation leading to unopposed estrogen and Hyperplasia

2. Polyp/ Fibroid /Adenomyosis

3. Cancer

Postmenopausal

1. Atrophy

2. Cancer/polyp

3. Estrogen therapy

4. Selective Estrogen Receptor Modulators

\section{Study Setting:-}

\section{Material And Methods}

The study conducted is a prospective hospital record based study in a tertiary health care centre-AcharyaVinobh aBhave Rural Hospital (AVBRH) attached to Jawaharlal Nehru Medical College (JNMC) which come under D 
attaMeghe Institute of Medical Sciences (DMIMS) University .The institute is situated in Sawangi (meghe) a vil lage about 4kms from Wardha. From September 2012 to september 2014.

2. Sample size- 100 subjects attending gynacopd with symptoms of AUB were taken for analysis

\section{Source Of Data:}

Patients admitted in Gynac ward of A. V. B. R.H. SawangiWardha, presenting abnormal uterine bleedi ng are included in our study by applying the following inclusion \& exclusion criteria. The study will be conduct ed during the period from September 2012 to September 2014.

Inclusion criteria

1. All OPD patients attending gynac clinic presenting with AUB

2. Age group between 21 to 60 years

Exclusion criteria

1. Pregnancy and pregnancy related conditions.

2. Females less than age 21 and more than 60 .

3. Patients with bleeding disorders.

4. Patients with anatomic causes.

\section{Method Of Collection Of Data}

(Including sampling procedure, if any):

Data will be collected from a specially designed Performa case recording Performa (CRF) pertaining to patient's particulars, proper history, clinical examinations, investigations, diagnosis \& surgical procedures. It is then subjected to statistical analysis with the help of biostatistician of our institute. All the surgical procedures \& medical management and investigations will be conducted under direct guidance and supervision of our guide. Before start of our study a written/informed consent will be obtained in local vernacular in each patient.

Statistical analysis

Statistical analysis was done by using descriptive and inferential statistics using $\mathrm{z}$ test for single proport ion. The software using analysis were SPSS 17.0 version and GRAPH PAD PRISM 5.01 version and $p<0.05$ is considered as level of significance.

The following analysis made are as follows:

1. Age distribution pattern(table no )

2. Relationship of AUB with Parity

3. Duration Of Abnormal Uterine Bleeding

4. Past history of study subjects

5. Family history of study subjects

6. Study Of Per Speculum Examination

7. Study Of Per Vagina Examination

8. Study Of Pap Smear

9. Abnormal Pap smear in study subjects

10. TVS ET $(\mathrm{mm})$ in the subjects studied

11. Bleeding patterns in AUB patients

12. Incidence of underlying structural causes in 100 subjects

13. Histopathological findings in the present study

14. Patterns of distribution of histopathological findings among various age groups.

15. Tables showing treatment and intervention done in study subjects

\section{Summary And Conclusion}

AUB is one of the most common problem in women of all age groups in reproductive period. It is chall enging gynecological problem caused by various endometrial pathologies. Endometrium is the mirror of hormon al status in women. Histological variations can be seen in the endometrium according to age of women and phas e of her menstrual cycle and any other specific pathology.

This was a study of a random selection of women from the gynac OPD in AcharyaVinobhaBhave Hosp ital who came with the complaints of Abnormal Uterine Bleeding. The women underwent ultrasonography and $\mathrm{e}$ ndometrial biopsy with all necessary investigations were performed and after full evaluation it was found that th e most common clinical presentation was Heavy Menstrual Bleeding followed by Intermittent Menstrual Bleedi ng. Maximum numbers of women came within 6 months of symptoms. The major risk factors observed were ag e, parity and menopausal status. The maximum incidence of AUB seen in patients in peri-menopausal age group i.e 36-40 years of age and those who were multiparous. 
With regards to diagnosis of AUB, pelvic ultrasound is the least invasive procedure used for used for vi sualization of the structure of the uterus and for visualization of the thickness of endometrium. In certain cases, $i$ $\mathrm{t}$ provides additional information that may assist both diagnosis and treatment choices.

Whereas Endometrial sampling could be effectively used as the first diagnostic step in abnormal uterin e bleeding although at times its interpretation could be quite challenging to the practicing pathologist. It is a sim ple, cost-effective and appropriate method that provides accurate diagnostic yield. The present study highlights $t$ he importance of endometrial biopsy and its interpretation which plays a pivotal role in the management of AUB . So the ultrasonography and endometrial biopsy still remains the gold standard for diagnosis of AUB in a rural s etup.

The treatment and intervention taken in 100 Study subjects in AVBRH are as follows: out of 100 study subjects 37\% had total abdominal hysterectomy, followed by therapeutic D\&C done in 29 subjects, Exploratoryl aprotomy was done in $16 \%$ and hormonal treatment given to $18 \%$ of cases.

Histopathological examination of endometrial biopsies in patients of abnormal uterine bleeding shows a wide spectrum of changes ranging from normal endometrium in various hormonal cycles to malignancy. In pre sent study, the most frequent finding seen in patients with AUB in there productive age group was proliferative phase. In peri and postmenopausal women simple hyperplasia without atypia was most frequently noted.

Further on post operative histopathology of specimens it was found that the prevalence of leiomyoma, adenomyosis and ovulatary disorders accounts for 55\% whereas endometrial polyp, endometrial stromal sarcom a were the rare findings in diagnosed cases of AUB in the present study. It was observed that AUB contribute $\mathrm{f}$ or a major bulk of gynac surgeries on going in AVBRH. That includes both major and minor procedures.

\section{Conclusions}

A study of 100 patients in clinically diagnosed with abnormal uterine bleeding was carried out from Se ptember 2012 to September 2014 in AVBRH.

Outcome of the Clinical and Histopathological findings are as follows:-

1. Patients belonging to various age groups were taken up for the study. The maximum incidence of AUB was in the 36-40 years age group. The minimum incidence of AUB was in 21-25 years.

2. With respect to parity of subjects, maximum incidence of abnormal uterine bleeding was seen in the parity of 1-3 and minimum incidence in nulliparous women.

3. The most common type of bleeding pattern observed was Heavy menstrual bleeding followed by intermenst rual bleeding in patients.

4. The maximum numbers of patients came with the 1-6 months duration of AUB. Hypertension was the com monest of the medical illnesses noted, followed by Diabetes mellitus. Severe Anemia was a coincidental fin ding in $7 \%$ of patients admitted with chronic AUB.

5. The role for pelvic ultrasound in the diagnosis of structural abnormalities is very important. In contrast In our study it was found that There are no pathognomonic sonographic characteristics that correlate completel y with histology, so comprehensive tissue diagnosis remains the gold standard.

6. The new classification system approved for the causes of AUB by International Federation of Gynecology a nd Obstetrics (FIGO) is PALM-COIEN: Polyp, adenomyosis, leiomyoma, malignancy and hyperplasia, coag ulopathy, ovulatory dysfunction, iatrogenic, endometrial causes and not yet classified.

The new terminologies have been approved by FIGO and they are :-

a) Heavy menstrual bleeding (HMB) should replace menorrhagia to describe excess of bleeding.

b) Inter menstrual bleeding (IMB) that occurs between clearly defined cyclic and predictable menses should re place the term metrorrhagia

c) Heavy and prolonged bleeding (HPB) should replace menometrorrhagia and frequent menstrual bleeding sh ould replace polymennorhea

In the present study, there were 40 cases of structural causes detected on clinical, ultrasonography and histopath ological examination of endometrial biopsies and $60(60 \%)$ cases belong to non structural causes.

7. The most common finding in the reproductive age group is proliferative endometrium whereas The simple hyperplasia without atypia was predominant finding in perimenopausal age group. Whereas atrophic endom etrium was mostly found in postmenopausal women.

8. In the present study, the two important observations were made regarding endometrial hyperplasia in AUB and they are:

a) Endometrial hyperplasia was highest in the age group of 41-50 years

b) It was highest in patients with history of heavy menstrual bleeding

9. All possible underlying causes60 were categorized according to new classification system. It was observed $t$ hat AUB contribute for a major bulk of gynac surgeries on going in AVBRH. That includes both major and minor procedures. 
10. AUB can be assessed by duration and timing of flow, haemoglobin or hematocrit, serum progesterone level $\mathrm{s}$, evaluation of the uterus on ultrasound testing, screening transvaginal ultrasound test of the endometrial ca vity and evaluation for coagulopathies with the use of structured history as screening.

11. In AVBRH, which is a rural hospital patients cuming with Abnormal Uterine Bleeding were analysed clinic ally and underwent ultasosnography and endometrial biopsy. The majority of patients has dual pathology wi th leiomyoma, adenomyosis and ovulatary disorders with predominating proliferative phase in histopatholo gy. Even though facilities are available for hormonal assays, CT and MRI, patients could not afford such co stly investigations. Hence ultrasound and endometrial biopsy still remains as Gold Standard for Rural patien ts.

In India, where majority is rural population, low cost investigations and treatment should be considered for the $b$ enefit of the patients. 